\title{
Electrical exploration of Massive Sulfides levels at Vermelho River, Digo-Digo Formation, Serra de Santa Rita Greenstone Belt, Goiás Velho, Brazil.
}

Pedro Augusto Costa do Amaral, Brenda Lorrana Borges Herdy, Hygor Vianna de Godoy, Arthur Chornobay, Moara Maria Oliveira de Matos, Celso Guerra Lemos, Victor José Cavalcanti Bezerra Guedes, Luan Machado Guimarães, Júlia Clícia Carvalho de Andrade Amorim, Catarina Labouré Bemfica Toledo e Welitom Rodrigues Borges (Instituto de Geociências Universidade de Brasília).

Copyright 2019, SBGf - Sociedade Brasileira de Geofísica

This paper was prepared for presentation during the $16^{\text {th }}$ International Congress of the Brazilian Geophysical Society held in Rio de Janeiro, Brazil, 19-22 August 2019.

Contents of this paper were reviewed by the Technical Committee of the $16^{\text {th }}$ International Congress of the Brazilian Geophysical Society and do not necessarily represent any position of the SBGf, its officers or members. Electronic reproduction or storage of any part of this paper for commercial purposes without the written consent of the Brazilian Geophysical Society is prohibited.

\section{Abstract}

This work presents the preliminary results of the use of electrical methods in the time domain to prospect VMS gold type, in the metavolcanics rocks of Digo-Digo formation, Serra de Santa Rita Greenstonebelt. The main objective is to identify subsurface structures and possible sulfidate zones. The acquisition of chargeability and resistivity data was realized with resistivity Syscal Pro 72 using the electrical tomography technique with dipoledipole array in the time domain. The resistivity and chargeability models reached 32.5 meters depth with RMS error of $57.1 \%$ and $7.2 \%$ respectively. The correlation of geological data and electrical models suggests three zones with different electrical properties. These zones were interpreted as chlorite- quartz schists, exhalative horizons (with massive sulfide levels) and metaultramafic rocks (Manoel Leocádio Formation). Two other small zones within exhalative horizons presented a high chargeability values $(40-70 \mathrm{mV} / \mathrm{V})$, these portions were interpreted as massive sulfide levels related to exposed levels in Digo-Digo and Vermelho River confluence.

\section{Introdução}

Uma ocorrência de ouro, associada a um depósito do tipo VMS, foi identificada no Greenstone Belt Serra de Santa Rita (GBSSR) na década de 1970 pela Metais de Goiás S.A. (METAGO), que realizou o primeiro programa de exploração na região.

$\mathrm{Na}$ época, a pesquisa mineral foi interrompida pelos baixos preços das comodities. Atualmente, a região está em fase inicial de pesquisa com trabalhos de exploração realizados pela empresa Orinoco Gold Ltda.

O prospecto localiza-se ao norte da cidade de Goiás Velho, associado a uma lente de metavulcanoclásticas félsicas do Membro Superior da Formação Digo-Digo com cerca de $17 \mathrm{~km}$ de comprimento, segundo NW, e 4 $\mathrm{km}$ de largura média, segundo NE (Jost et al.,2014).

Os métodos elétricos são efetivos na identificação de alvos VMS pois respondem à condutividade elétrica das rochas e minerais, que pode variar em até 20 ordens de grandeza (Grant e West, 1965).

Estes depósitos têm alta condutividade, superiores a 500 $\mathrm{mS} / \mathrm{m}$ (Ford, 2007). Comparado com rochas ígneas e metamórficas com condutividades típicas de $<1 \mathrm{mS} / \mathrm{m}$ e rochas sedimentares com condutividades de 1 a 500 $\mathrm{mS} / \mathrm{m}$, o contraste entre depósitos VMS e sua rocha hospedeira pode ser significativo sendo assim uma propriedade física útil (Morgan, 2013).

O objetivo da presente pesquisa é a investigação preliminar da estrutura e posicionamento em subsuperfície dos níveis de sulfeto maciço expostos nas margens da confluência do córrego Digo-Digo e Rio Vermelho, com uso de métodos elétricos (resistividade e polarização induzida) no domínio do tempo.

\section{Contexto Geológico}

O GBSSR, está situado na porção sul do Terreno Arqueano de Goiás, é limitado pelos complexos granitognáissicos Caiçara (a norte) e Uvá (a sul). Apresenta trend estrutural com orientação $\mathrm{N} 50^{\circ}-70^{\circ} \mathrm{W}$, é separado a noroeste do Greenstone Belt de Faina pela falha de Faina, com movimentação dextral e direção $\mathrm{N} 30^{\circ} \mathrm{E}$ (Resende et al., 1998; Figura 1).

A porção basal do GBSSR é constituída por rochas vulcânicas de composição ultramáfica que caracterizam a Formação Manoel Leocádio, seguidas por rochas vulcânicas de composição máfica da Formação DigoDigo, que contem lentes de rochas vulcânicas de composição intermediária a félsica no topo (Resende et al., 1998). A sequência vulcânica basal possui idade de 2.94 $\pm 0.02 \mathrm{Ga}$ (Borges et al., 2017) e é recoberta uma sequência sedimentar formada por metacherts, calcixistos, formações ferríferas bandadas da Formação Limeira e metagrauvacas interpretadas como sequências turbidíticas da Formação Fazenda Cruzeiro (Resende et al., 1998).

A Formação Digo-Digo constitui-se de uma sucessão de rochas vulcanoclásticas subaquosas de composição andesítica a riodacítica. Diques e stocks de diorito e riolito ocorrem em meio às vulcanoclásticas. horizontes exalativos métricos compostos de metacherts bandados com filitos carbonosos, ricos em pirita e ouro, ou de intervalos de pirita maciça foram identificados nas margens do córrego Digo-Digo (Resende et al. 1999, Jost et al. 2014). 


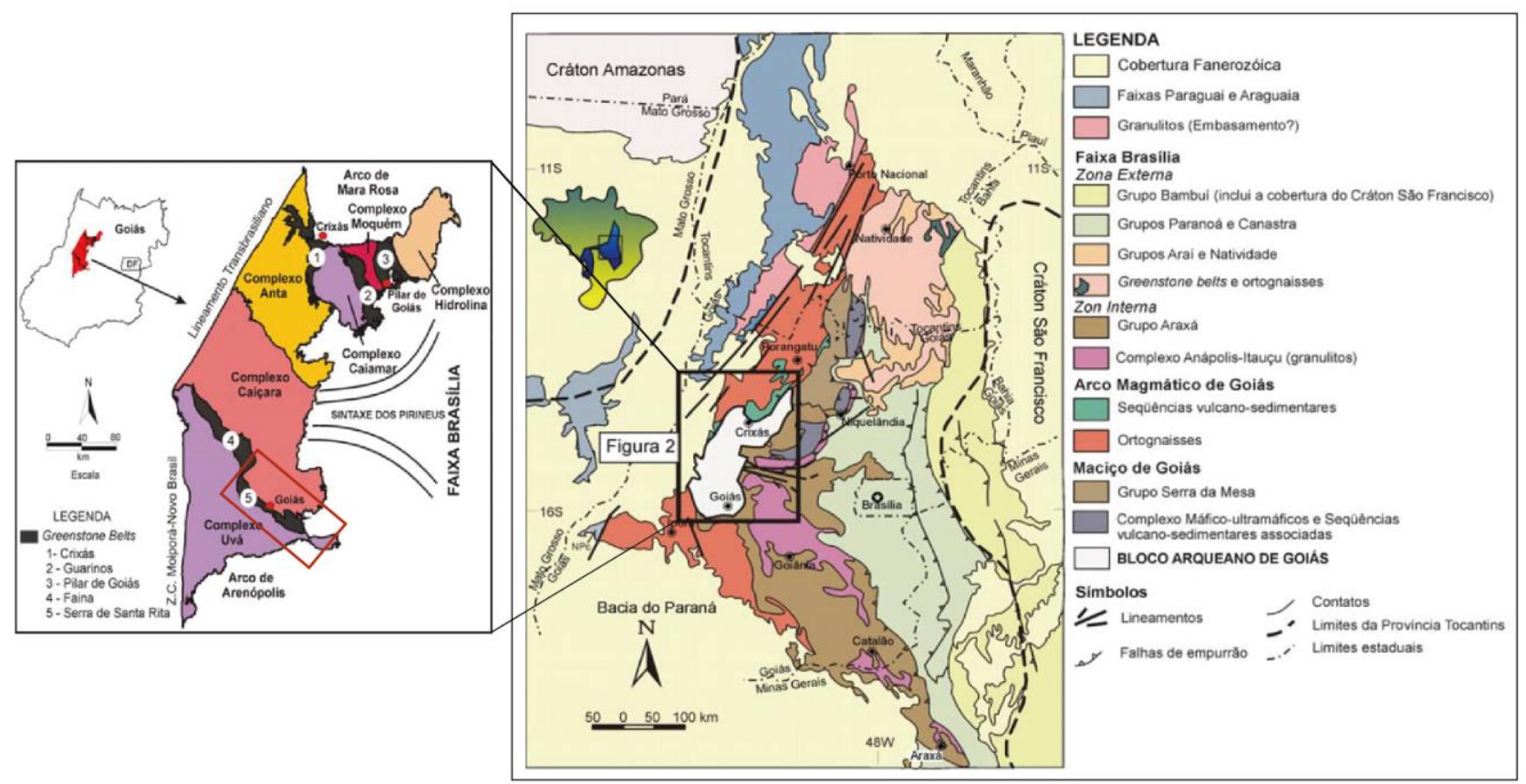

Figura 1 - Mapa Geológico da porção central do Brasil com bloco arqueano de goiás delimitado (á direita; modificado de Pimentel et al.,2000). Mapa geológico do Bloco Arqueano de Goiás com Greenstone Belt Serra de Santa Rita delimitado em vermelho (à esquerda; modificado de Jost et al., 2014).

\section{Área de Estudo}

O objeto de estudo está inserido no extremo sudoeste do Greenstonebelt Serra de Santa Rita, na porção centro norte da Formação Digo-Digo, próximo ao contato com as rochas meta-ultramáficas da Formação Manoel Leocádio, que estão representadas na área majoritariamente por talco xistos (Fig. 2). Nessa porção, as rochas da Formação Digo-Digo presentes correspondem ao topo da sequência meta-vulcânica (Resende et al., 1998), identificadas em campo principalmente como: clorita-quartzo xistos, metavulcânicas, vulcanoclásticas e intercalações de filitos carbonosos e metacherts com ocorrências de níveis de pirita maciça (horizontes exalativos). $\mathrm{Na}$ área as rochas possuem foliação principal com mergulhos moderados $\left(40-60^{\circ}\right)$ para sudoeste. 


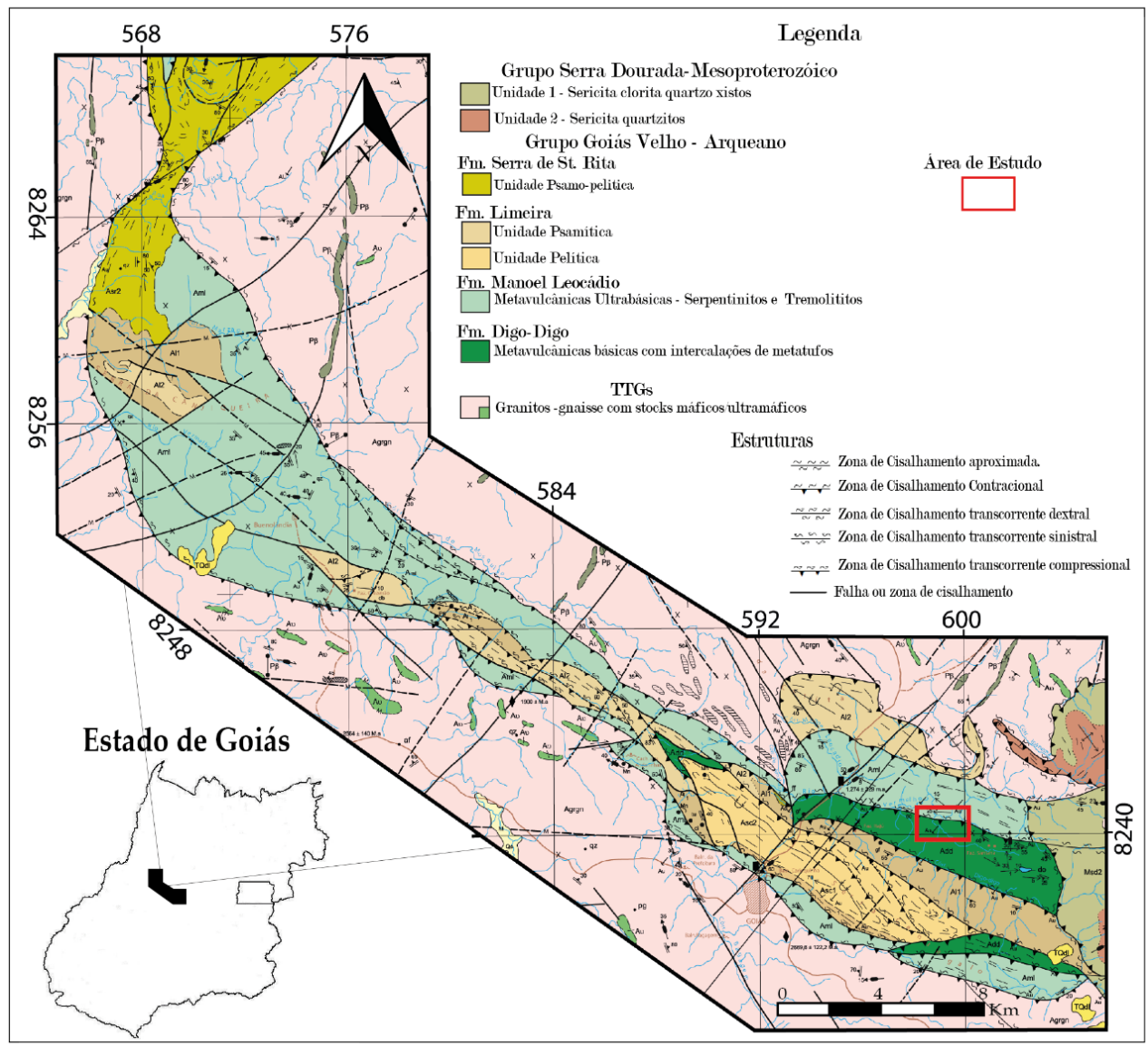

Figura 2 - Mapa Geológico na escala 1:100.000 do Greenstone Belt Serra de Santa Rita, com área de estudo delimitada (modificado de Baeta, 2000).

\section{Aquisição dos Dados}

$\mathrm{Na}$ aquisição dos dados 2D de resistividade e polarização induzida (DC/IP) escolheu-se o método da tomografia elétrica com o arranjo Dipolo-Dipolo em função de sua maior resolução e sensibilidade na delimitação de estruturas inclinadas. No campo optou-se pelo posicionamento da seção 2D com 530 metros de extensão (fig. 3), paralela à região de ocorrência de afloramento do horizonte exalativo associado a sulfetos maciços da Formação Digo-Digo e semi-perpendicular a direção das estruturas geológicas regionais.

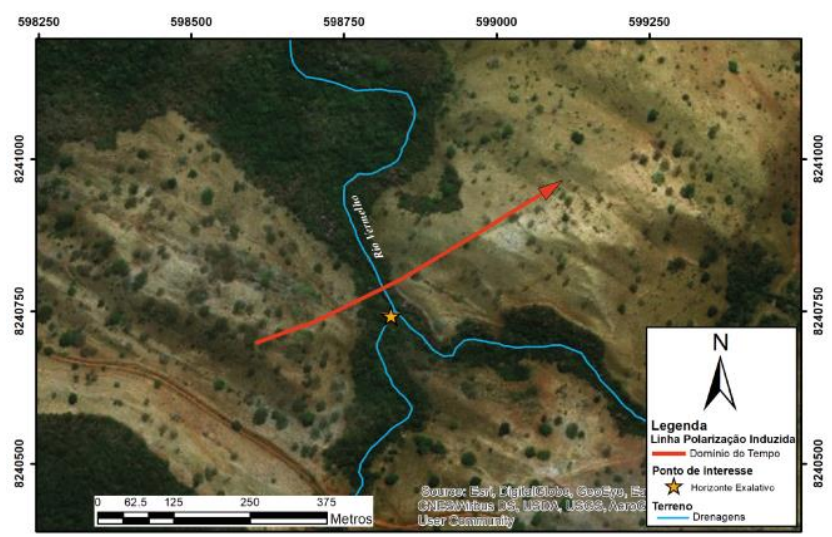

Figura 3 - Mapa de localização da linha de aquisição elétrica e da ocorrência de sulfetos maciços. 
A aquisição dos dados elétricos ocorreu com uso do resistivimetro multi-eletródico SYSCAL PRO 72 (Iris Instruments). No protocolo de aquisição programaram-se voltagem de $800 \mathrm{~V}$, tempo de injeção de corrente de $2 \mathrm{~s}$, e amostragem de 2 (mínima) a 3 (máxima) medidas por ponto. No campo usaram-se eletrodos não polarizáveis nos pontos de registro de potencial elétrico, e eletrodos de aço carbono nos pontos de injeção de corrente elétrica (fig.4).

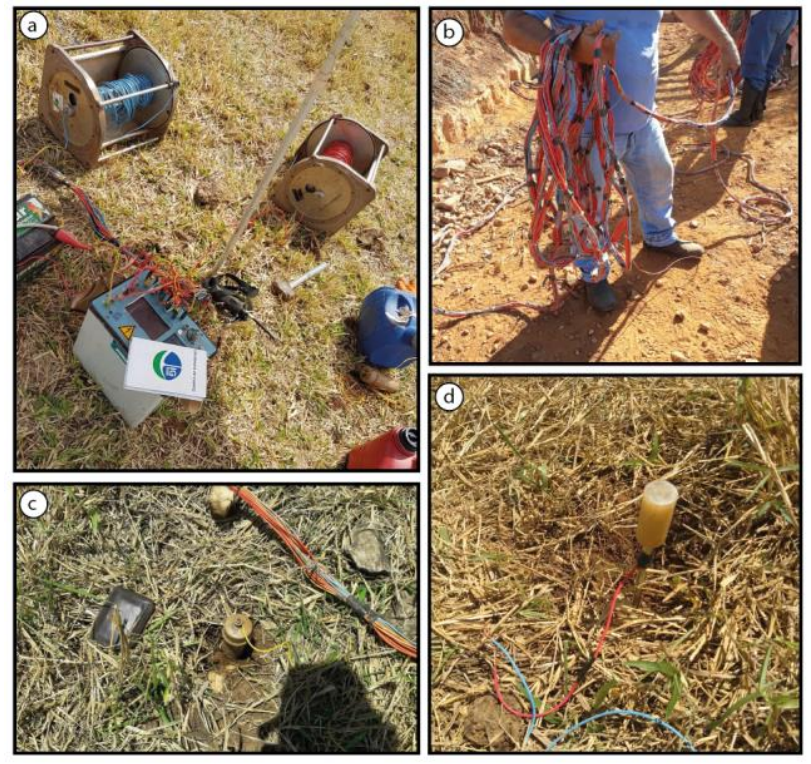

Figura 4- a) Resistivimetro Syscal Pro 72. b) Cabos utilizados na aquisição. c) Eletrodo não polarizável (potencial). d) Eletrodo de aço carbono (injeção de corrente).

\section{Processamento dos Dados}

No software Prosys II (Iris Instruments), após a inserção da topografia realizou-se a filtragem dos dados de resistividade e polarização induzida separadamente.

$\mathrm{Na}$ filtragem dos dados de resistividade, transformou-se os valores de rho em absolutos, filtrou-se destes valores os menores que 2 e maiores que 10.000 , além de valores discrepantes no espectro de resistividade. Para os dados de IP, retirou-se valores negativos e superiores a $90 \mathrm{mV} / \mathrm{V}$ e valores incoerentes com a curva de decaimento do efeito IP.

No software Res2Dinv realizou-se em ambos os dados, uma segunda filtragem com uso da ferramenta "exterminate bad datum points". A rotina de inversão deuse no mesmo programa.

$\mathrm{Na}$ inversão dos dados optou-se pela a inversão robusta que tende a destacar estruturas verticalizadas. Nos modelos de cargabildade e resistividade utilizou-se a escala linear e logarítmica, respectivamente.

\section{Resultados}

Os modelos de resistividade (fig. 5a) e cargabilidade (fig. $5 b)$ foram obtidos em 4 iterações, atingiram 32,5 metros de profundidade de investigação, com erros RMS de $57,1 \%$ e $7,2 \%$ respectivamente.

Na posição entre 250 e 420 metros, há presença de altos valores de cargabilidade com valores entre 50 e $95 \mathrm{mV} / \mathrm{v}$, estas anomalias possuem posições coerente com a da ocorrência de horizontes exalativos com níveis de sulfeto maciço presentes na confluência do córrego Digo-Digo e Rio Vermelho.

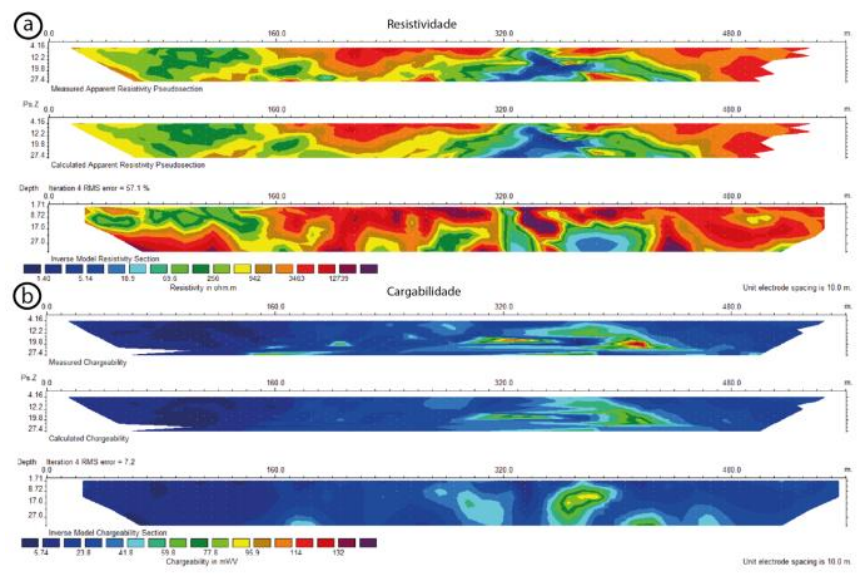

Figura 5 - a) Modelos de resistividade medida, aparente e resultado da inversão. b) Modelos de cargabilidade medida, calculada e resultado da inversão.

\section{Discussões e Conclusões}

A partir da correlação dos dados geológicos e geofísicos foi possível a elaboração de um modelo geológico da linha (Figura 6).

Nos modelos elétricos, a região entre 0 e 180 metros foi interpretada como os clorita-quartzo xistos da Formação Digo-Digo, apresentaram os menores valores de cargabilidade da seção (1 a $10 \mathrm{mV} / \mathrm{V}$ ).

Na porção central da seção, entre aproximadamente 180 e 410 metros, a região foi correlacionada ao horizonte exalativo com metavulcânicas intermediárias subordinadas, unidade onde estão presentes os níveis de sulfeto maciço expostos no córrego. As anomalias positivas de cargabilidade com valores de 40 a $70 \mathrm{mV} / \mathrm{V}$ nessa região foram interpretadas como a possível continuidade dos níveis sulfetados.

O restante da seção entre 410 e 560 metros, apresentou valores de cargabilidade intermediários de $10 \mathrm{a} 28 \mathrm{mV} / \mathrm{V}$, e os maiores valores de resistividade da seção, esta região foi correlacionada as rochas metaultramáficas da Formação Manoel Leocádio. 

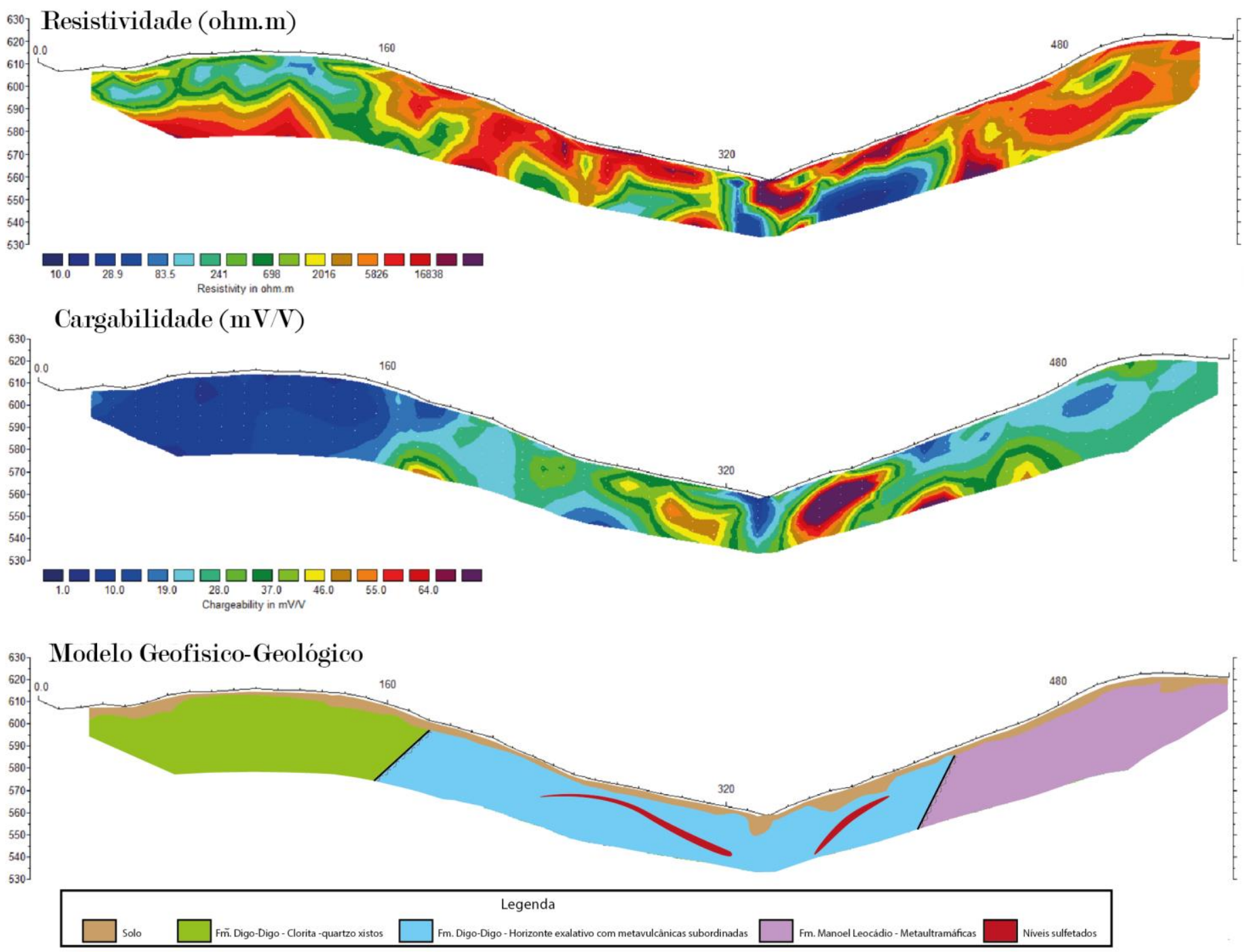

Figura 6 - Modelos de resistividade, cargabilidade e geofísico-geológico.

\section{Agradecimentos}

Agradecemos aos funcionários da Orinoco Gold: Cicinho, Lucimar, Romes e Lucas de Jesus pelo auxílio em campo. Aos geólogos: Thiago Vaz, Guilherme Texeira e Samuel Machado pelo auxilio nas etapas pré-campo.

Agradecemos aos alunos da disciplina de Métodos Elétricos (2/2018) do curso de Geofísica da Universidade de Brasília, pelo árduo trabalho realizado na coleta dos dados desta pesquisa.

O presente trabalho foi realizado com apoio da Coordenação de Aperfeiçoamento de Pessoal de Nível Superior - Brasil (CAPES) - Código de Financiamento 001.

\section{Referências}

Baêta Júnior, J.D.A., Oliveira, C. C., Pinheiro, M. M., Andrade, R. S., Camargo, M. A., 2000. Programa Levantamentos Geológicos Básicos do Brasil - Escala 1:100,.000, Folha SD.22-Z-C-V, Goiás. CPRM.

Ford, K., Keating, P., and Thomas, M.D., (2007), Overview of geophysical signatures associated with Canadian ore deposits, in Goodfellow, W.D., ed., Mineral deposits of Canada-A synthesis of major deposit-types, district metallogeny, the evolution of geological provinces, and exploration methods: Geological Association of Canada, Mineral Deposits Division, Special Publication 5, p. 939-970. 
Grant, F.S., and West, G.F., 1965, Interpretation theory in applied geophysics: New York, McGraw-Hill Books Company, p. 584.

Jost, H., Carvalho, M.J., Rodrigues, V.G., Martins, R. 2014. Metalogênese dos greenstone belts de Goiás. In: Silva, M.G., Neto, M.B.R., Jost, H., Kuyumjian, R.M. (Orgs.), Metalogênese das províncias tectônicas brasileiras, Belo Horizonte, CPRM, p. 141-168.

Pimentel M.M., Jost H., Fuck R.A. 2004. O embasamento da Faixa Brasília e o Arco Magmático de Goiás. V. Mantesso-Neto, A. Bartorelli, C.D.R. Carneiro, B.B.B. Neves (Org.) Geologia do Continente Sul-Americano: evolução da obra de Fernando Fávio Marques de Almeida. Beca Produções Culturais Ltda., São Paulo, pp.: 356-368

Resende M.G., Jost H., Osborne G. A., Mol A G. 1998. Stratigraphy of the Goiás and Faina greenstone belts, Central Brazil: a new proposal. RBG, 28, p. 77-94.

Resende M.G., Jost H., Lima B.E.M., Teixeira A.A. 1999. Proveniência e idades-modelo $\mathrm{Sm}-\mathrm{Nd}$ de rochas siliciclásticas arqueanas dos greenstone belts de Faina e Santa Rita, Goiás. RBG, 29, p.281-290. 